\title{
Physiological synaptic signals initiate sequential spikes at soma of cortical pyramidal neurons
}

\author{
Rongjing Ge, Hao Qian and Jin-Hui Wang*
}

\begin{abstract}
The neurons in the brain produce sequential spikes as the digital codes whose various patterns manage wellorganized cognitions and behaviors. A source for the physiologically integrated synaptic signals to initiate digital spikes remains unknown, which we studied at pyramidal neurons of cortical slices. In dual recordings from the soma vs. axon, the signals recorded in vivo induce somatic spikes with higher capacity, which is associated with lower somatic thresholds and shorter refractory periods mediated by voltage-gated sodium channels. The introduction of these parameters from the soma and axon into NEURON model simulates sequential spikes being somatic in origin. Physiological signals integrated from synaptic inputs primarily trigger the soma to encode neuronal digital spikes.
\end{abstract}

Keywords: action potential soma, axon, refractory period, sodium channels

\section{Introduction}

The neurons are one of basic units to fulfill the brain functions, and their events are executed at different subcellular compartments, such as the reception of synaptic inputs, the integration of these synaptic signals, the production of action potentials and the secretion of neurotransmitters [1,2]. In terms of the sources for firing action potentials, the current belief is that action potentials are generated at axon hillock [3-11]. In these studies, short-time square pulses are given and a single spike is induced. However, the regulations and mechanisms for the physiological signals integrated from synaptic inputs to trigger the spikes remains unknown.

The neurons integrate the signals from numerous synapses and produce sequential spikes as the digital codes to carry various messages under the physiological conditions $[12,13]$. These integrated signals in vivo are long-time in nature, and their depolarization pulses induce sequential spikes [14-18] and Figure 1). A source for these in vivo signals to initiate sequential spikes has not been documented, which we have investigated at cortical pyramidal neurons by dual- recording their soma and axonal bleb simultaneously.

* Correspondence: jhw@sun5.ibp.ac.cn

State Key Lab for Brain and Cognitive Sciences, Institute of Biophysics,

Chinese Academy of Sciences, Beijing, China 100101

\section{Results}

Integrated synaptic signals are long-time pulses in patterns of steady-state and fluctuation

The physiological sources of firing action potentials are ideally identified by using in vivo signals, which has not been documented yet. In order to address this issue, we have analyzed these signals that were intracellularly recorded from cortical pyramidal neurons in living mice.

In vivo signals including those inducing sequential spikes (Figure 1A) and subthreshold pulses (Figure 1B) appear long time. Figure $1 \mathrm{C}$ illustrates that these depolarization pulses integrated in vivo fall into a range of 50 1600 ms in their durations. These in vivo signals are generally classified into steady-state pulses (an extended waveform in left panel of Figure 1B) and fluctuation ones (in right). The former is similar to direct-current pulses used to induce spikes in the most of electrophysiological experiments, and the latter is simulated as a cosine model [19]. The percentages of steady-state forms and fluctuation ones in these in vivo signals are approximately $61 \pm 5 \%$ and $39 \pm 5 \%$, respectively (Figure $1 \mathrm{D}, \mathrm{n}=11$ cells). Therefore, the physiological signals to induce sequential spikes are long-time depolarization pulses, which we used to identify the sources of sequential spikes. 

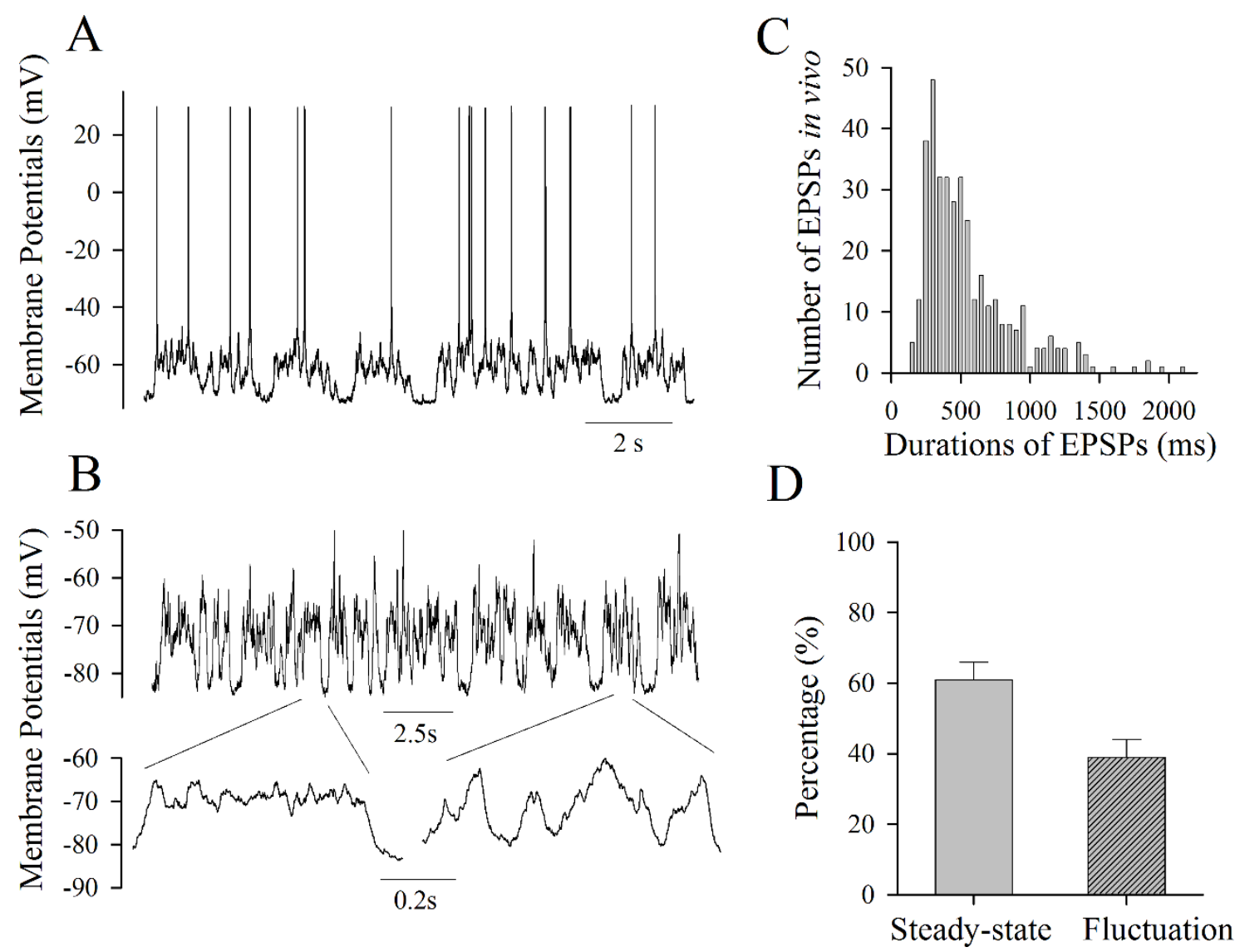

Figure 1 In vivo depolarization signals for spike initiation and subthreshold are long-time in nature. A) The integrated synaptic signals induce spikes. B) shows the integrated synaptic signals at subthreshold level (top panel) and the expanded waveforms (bottoms), which appear steady-state pattern (square pulse) and fluctuation one (cosine). C) shows number of EPSPs in vivo vs. signal durations, which fall into a range of 50 1600 ms. D) illustrates the percentages for steady-state pattern and fluctuation one analyzed from total in vivo signals ( $n=11$ neurons).

\section{Physiological synaptic signals induce sequential spikes more efficiently at the soma than axon}

In theory, no matter what the axon or soma is a primary site to encode sequential spikes, it should have the higher ability to convert analogue input signals into digital spikes, i.e., more efficient input- output. The soma and axonal bleb (20 45 $\mu \mathrm{m}$ away from the soma) of identical pyramidal neurons in neocortical slices were recorded simultaneously in whole-cell current-clamp (inset in Figure 2A). The in vivo signals (bottom trace in Figure $2 \mathrm{~A}$ ) in various intensities were injected into these two locations, respectively, to assess their input-output couplings. These long-time pulses induced more spikes at the soma (red trace in Figure 2A) than the axon (blue). Figure 2B shows spike number vs. normalized pulses in vivo at the soma (red triangles) and axon (blue circles, $n=19$ ), in which somatic input-output curve is on the top side of axonal one. The facts that somatic spike thresholds are lower and identical stimuli induce more spikes at the soma indicate a somatic origin of firing sequential spikes.
If the soma encodes spikes, it should have the highest ability of firing spikes in response to inputs, like a principle that sinoatrial node in the highest rate controls heart beat. Figure $2 \mathrm{C}$ shows input-output curves at the soma (red symbols, $\mathrm{n}=27$ ) and axonal segments in 10 30 $\mu \mathrm{m}$ (axon hillock; blue, $\mathrm{n}=15$ ) and $>30 \mu \mathrm{m}$ (green, $\mathrm{n}=12$ ) away from the soma i.e., a decreasing trend in the ability of firing spikes from the soma to the axon. This result indicates that the soma is more sensitive to long-time signals and dominantly produces spikes. The physiological in vivo signals induce sequential spikes primarily at the soma of cortical pyramidal neurons.

\section{Latencies between somatic spikes and axonal ones favor a somatic origin of spike initiation}

The initiation of spikes was defined at a time point of minimal $d v / d t$ but larger than zero (Figure $3 \mathrm{~A} \sim \mathrm{B}$ and Methods). Latencies between somatic spikes and axonal ones $(\Delta \mathrm{T})$ were calculated. Figure $3 \mathrm{C}$ shows that $\Delta \mathrm{T}$ values for spikes $1 \sim 3$ are $-2.5 \pm 87,-311 \pm 226.5$ and $-471 \pm 215.8 \mu \mathrm{s}(\mathrm{n}=20)$, respectively. In spite of big 

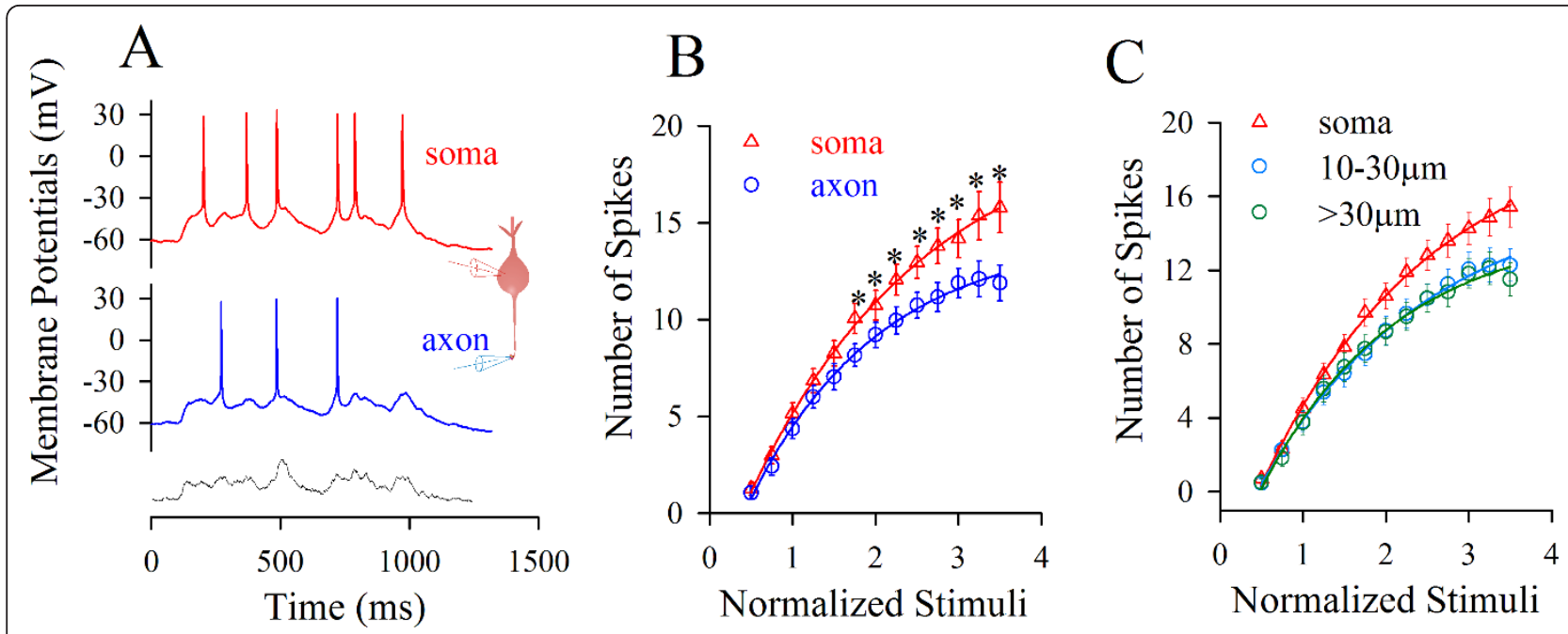

Figure 2 In vivo signals initiate sequential spikes preferentially at the soma of cortical pyramidal cells. A) An in vivo pulse (black trace at bottom, >1000 ms) evokes more spikes at the soma (red trace) than at axon (blue) in dual recording (inset). B) shows spike number vs. normalized pulses in vivo at the soma (triangle symbols) and axon (circles; $n=19$; asterisks, $p<0.05$ ). C) illustrates input-output curves for the soma (red symbols) and different axonal fragments in 10 30 $\mu \mathrm{m}$ (blue) and $>30 \mu \mathrm{m}$ (green) away from the soma.

variation for $\Delta \mathrm{T}$ values, sequential spikes recorded at the soma appear ahead of those at the axon.

It is noteworthy that we validated the approach used to localize the time point of spike initiation in Figure $3 \mathrm{~A} \sim \mathrm{B}$. If the time point of minimal $d v / d t$ is a good measurement to locate spike onset mediated by voltagegated sodium channels (VGSC), it should be associated with spikes, and the manipulation of VGSCs should affect this point and spikes proportionally. In other words, if VGSCs are partially blocked, we should see the proportional changes of its time phases with spike amplitudes, as well as observe the presence of this point and the spikes with a loss of the initiation sites that were previously defined [20-24].

Based on analyzing the amplitudes of spike $d v / d t$ and the time of minimal $d v / d t$ to its peak in the presence of QX-314 that is an inhibitor of VGSCs (0.5 mM intracellularly in Figure 3D), we observed the proportional correlation between these two parameters (36 spikes from 3 cells in Figure 3E) under the condition of partial VGSCs' inactivation. Moreover, spike initiation defined previously (purple dots in $3 \mathrm{D}$ and $3 \mathrm{~F}$ ) is located between minimal $d v / d t$ site and spike fast-rising phase, which divides the spike rising-phase into two components exponentially (blue trace in 3F). Figure 3F shows that $d v / d t$ values of spike rising-phase are better fitted into two exponentials under control $\left(\mathrm{r}^{2}=0.99\right)$ and an exponential $\left(r^{2}=0.99\right)$ under partial VGSC inactivation. A loss of spike-onset site previously defined indicates that the previous site is not always associated with spikes. Taken these data together, we conclude that the minimal $d v / d t$ site is better for representing spike initiation.
VGSC-mediated spike thresholds and refractory periods are lower at the soma than axon

In terms of mechanisms for physiological signals to primarily induce somatic spikes, we propose that long-time depolarization makes somatic spike thresholds and refractory periods lower than axonal ones. Thresholds and refractory periods were measured by injecting depolarization pulses $(50 \mathrm{~ms}$, a minimal duration of in vivo signals in Figure 1, and Methods) into the soma and axonal bleb of identical neurons, respectively [14,25]. A pulse induced a spike at the soma (red trace in Figure $4 \mathrm{~A}$ ) but not the axon (blue). Figure $4 \mathrm{~B}$ illustrates that somatic threshold $(122.5 \pm 10 \mathrm{pA}$, red bar $)$ is lower than axonal one $(152 \pm 12 \mathrm{pA}$, blue; $\mathrm{p}<0.05 ; \mathrm{n}=12)$.

Moreover, paired-pulses (50 ms for their duration) were used to measure the refractory periods of somatic spikes (red traces in Figure 4C) and axonal ones (blues). Figure 4D shows that somatic refractory periods (red bar) and axonal ones (blue) are $95.5 \pm 17.6$ and $141.7 \pm$ $19.3 \mathrm{~ms}$, respectively $(\mathrm{p}<0.05 ; \mathrm{n}=9)$. When long-time depolarization signals are integrated at pyramidal cells, somatic spike thresholds and refractory periods are lower than axonal ones, which make sequential spikes primarily induced at cell body.

To the mechanism underlying lower somatic thresholds and refractory periods by long-time pulse, we propose that long-time depolarization partially inactivates axonal voltage-gated sodium channels (VGSC). If it is a case, we should see the prevention of VGSC inactivation lowers axonal thresholds and refractory periods. Anemone toxin (ATX) that attenuates VGSC inactivation [26] was selected to prevent axonal VGSC inactivation. 


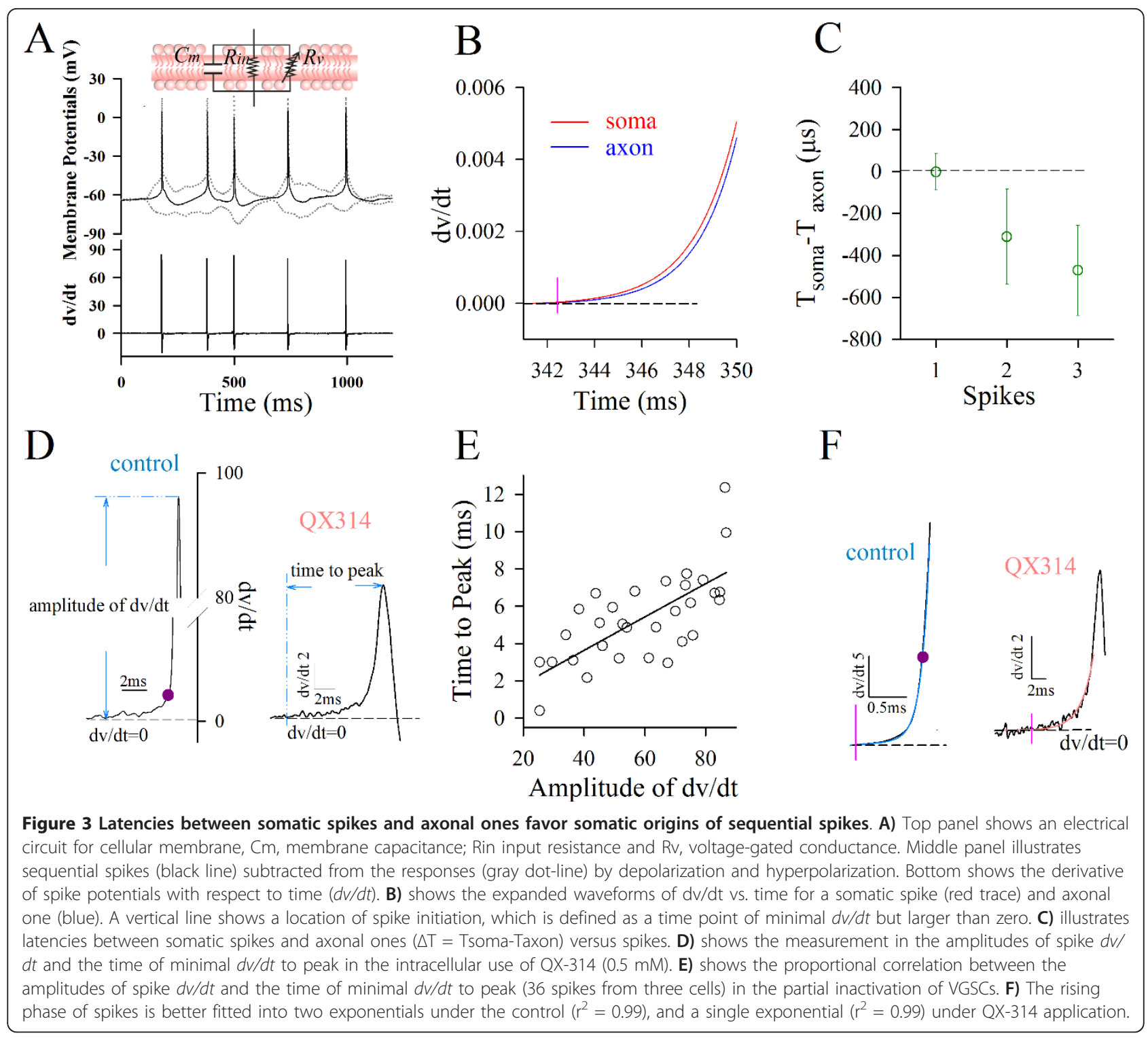

We measured thresholds and refractory periods of axonal spikes induced by depolarization pulses $(50 \mathrm{~ms})$ during applying $5 \mu \mathrm{M}$ ATX onto axonal blebs. ATX appears to reduce axonal spike thresholds (green traces in Figure 5A) and refractory periods (green in Figure $5 \mathrm{~B})$. Figure $5 \mathrm{C} \sim \mathrm{D}$ illustrate spike thresholds and refractory periods before (blue bars) and after ATX application (greens; $\mathrm{p}<0.05, \mathrm{n}=9$ ), respectively. The results indicate that long-time depolarization increases axonal thresholds and refractory periods through inactivating local VGSCs.

\section{Lower thresholds and refractory periods at the soma simulate a somatic origin of spikes}

The results above indicate that long-time signals mainly inactivate axon's VGSCs and lead to the high values of spike thresholds and refractory periods. As a result, physiological signals initiate spikes being somatic in origin. To strengthen this point, we introduced the factors from the axon and soma into NEURON [27,28]. In vivo signals induced more simulated spikes at the soma (red trace in Figure 6AE) than the axon (blue). The thresholds of spikes are lower and the number of spikes by identical stimuli is higher at the soma (red symbols in Figure 6B) than the axon (blue). The result from computational simulation is consistent with those from experiments (Figure 2).

\section{Discussion}

Long-time in vivo signals induce sequential spikes at the soma of cortical pyramidal neurons (Figure 2). In terms of the mechanism, these pre-depolarization signals 


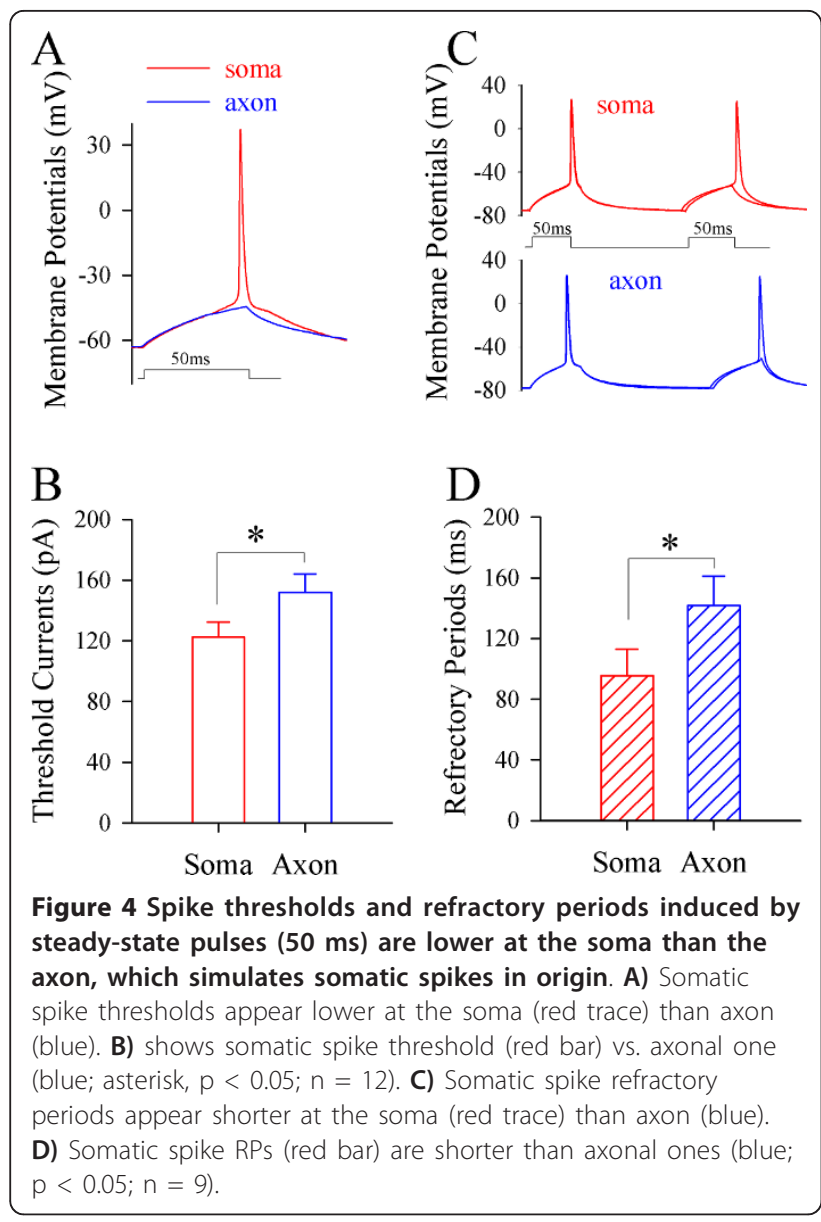

mainly inactivate axonal VGSCs (Figures 4 5), such that somatic thresholds and refractory periods are lower (Figure 4), and the sequential spikes are somatic in origin (Figure 6). Our data from the experiments and computational simulation favor a somatic source for physiological signals to induce digital spikes, which updates the knowledge that an action potential is only initiated at the axonal hillock. Our data also supports a hypothesis that spikes may be generated on dendrites or soma in other types of neurons [29-36].

Short-time pulses initiate a single spike at axon hillock $[3,6,8]$, which is supported by low thresholds and high density of VGSCs at this segment [4,5,9-11,37-48], and axonal spikes ahead of somatic ones [20-24]. The major reasons for this belief versus our findings include the followings. Previous studies used short-time pulses (less than $5 \mathrm{~ms}$ ) to induce a single spike, but we applied long-time depolarization signals that fall into the range of the durations of integrated synaptic inputs (Figure 1C) to induce sequential spikes. More importantly, the pulses used in our studies are the in vivo signals (Figures $1,2,3$ ), which should be more physiological.
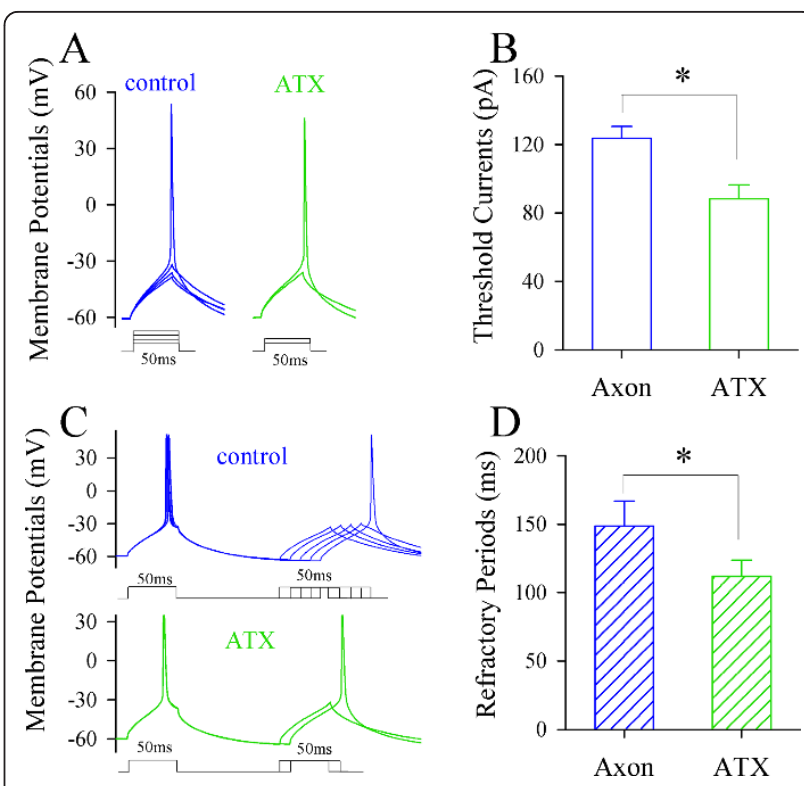

Figure $\mathbf{5}$ The prevention of axonal VGSC inactivation by ATX decreases axonal spike thresholds and shortens refractory periods. A) shows the waves of axonal spike thresholds induced by depolarization pulses (50 ms) before and after ATX application (5 $\mu \mathrm{M}$, green trace). B) shows the averaged values of axonal spike thresholds before and after ATX application. C) shows the waveforms of axonal spike refractory periods induced by depolarization pulses (50 ms) before and after ATX application (5 $\mu \mathrm{M}$, green trace). D) shows the averaged values of axonal spike refractory periods before and after ATX application.

We do observe that short-time pulses make axonal threshold and refractory period low, and evoke an axonal spike. However, the signals in vivo are above $50 \mathrm{~ms}$ (Figure 1), and are integrated from unitary synaptic events that last for longer than $20 \mathrm{~ms}[13,14]$. Longtime pulses make somatic thresholds and refractory periods to be lower than axonal ones (Figure 4), such that physiological signals induce sequential spikes being somatic in origin. In this regard, there may be a plasticity of spike-onset location that depends on the patterns of synaptic inputs, as we have observed.

VGSC densities are high at the remote side of axonal hillock such that computational simulations show an axonal origin of spike initiation $[4,9,10,45,46]$. However, the number of functional VGSCs is not higher at axonal hillock [49]. Axonal VGSCs may be easily inactivated and less reactivated by long-time pulses (Figures 4 5). Therefore, the initiation of sequential spikes depends on the functional states of VGSCs, instead of their higher densities. One could argue the results that sodium imaging indicates high functional VGSCs' density at AIS $[20,22,50]$. As somatic volume is much larger than axonal one, the quantity of $\mathrm{Na}^{+}$influx during firing spikes is buffered substantially at the soma. Less change in the somatic $\mathrm{Na}^{+}$signal may not reflect a low level of 

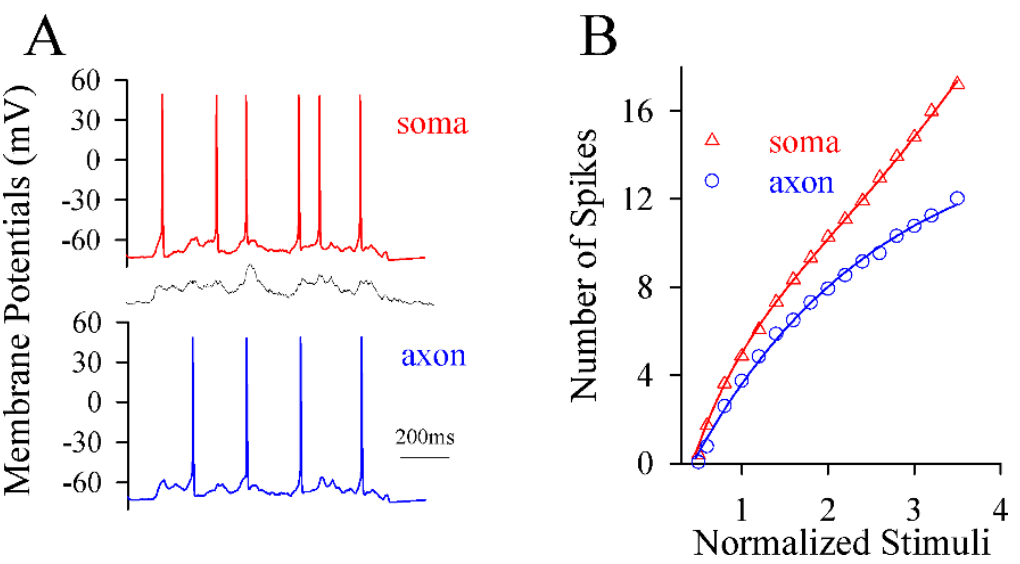

Figure 6 Computational simulation based on spike thresholds and refractory periods at the soma and axon favors the sequential spikes being somatic in origin. A) The number of spikes in computational simulation is higher at the soma (red trace) than the axon (blue) induced by in vivo signal (middle). B) illustrates the number of spikes vs. the intensity of stimulus pulses at the soma (red triangle symbols) and the axon (blue circles).

functional VGSCs and a slow initiation of spikes at the soma.

A measurement of latencies between axonal spikes and somatic ones indicates the origin of spike initiation at AIS [20-24]. In such studies, the effect of passive membrane property on measuring temporal signals was present, and spike initiation was defined at various time points. With reducing the effect of membrane property and defining minimal $d v / d t$ as a site of spike initiation that is always associated with spikes (Figure 3), we found that the latency between somatic spikes and axonal ones favors somatic spikes in origin.

What is physiological significance for higher dense VGSCs at axonal hillock? It may facilitate somatic spikes propagating toward axonal terminals [14]. Moreover, the nearby end of AIS is innervated by GABA synapses [51] and has less VGSCs $[9,10,43]$. The higher dense VGSCs at remote AIS, while GABA synapses induce a hyperpolarization, compensate loss of signals at nearby AIS, a homeostasis between subcellular compartments [12].

What is the physiological significance for the somatic source of sequential spikes? If the soma integrates input signals to initiate spikes at the axon, these integrated signals should be propagated to the axon. That long-time depolarization signals inactivate axonal VGSCs and GABAergic synapses at AIS shut these signals will weaken this propagation. To prevent any potential dissociation between the integration of synaptic signals and the conversion of them into digital spikes, these two processes are better fulfilled in a single unit, i.e., the soma.

\section{Materials and methods}

\section{Brain slices}

Cortical slices $(300 \mu \mathrm{m})$ were prepared from FVB-Tg (GadGFP)45704Swn/J mice. The mice in postnatal days
15-20 were anesthetized by injecting chloral hydrate $(300 \mathrm{mg} / \mathrm{kg}$ ) and decapitated with a guillotine. The slices were cut with a Vibratome in the modified and oxygenized (95\% $\mathrm{O}_{2} / 5 \% \mathrm{CO}_{2}$ ) artificial cerebrospinal fluid (mM: $124 \mathrm{NaCl}, 3 \mathrm{KCl}, 1.2 \mathrm{NaH}_{2} \mathrm{PO}_{4}, 26 \mathrm{NaHCO}_{3}, 0.5$ $\mathrm{CaCl}_{2}, 5 \mathrm{MgSO}_{4}, 10$ dextrose and 5 HEPES; pH 7.35) at $4^{\circ} \mathrm{C}$, and then were held in the normal oxygenated ACSF (mM: $126 \mathrm{NaCl}, 2.5 \mathrm{KCl}, 1.2 \mathrm{NaH}_{2} \mathrm{PO}_{4}, 26$ $\mathrm{NaHCO}_{3}, 2.0 \mathrm{CaCl}_{2}, 2.0 \mathrm{MgSO}_{4}$ and 25 dextrose; $\mathrm{pH}$ $7.35) 35^{\circ} \mathrm{C}$ for 1 hour before the experiments. A slice was transferred to a submersion chamber (Warner RC26G) that was perfused with normal ACSF for electrophysiological experiments [52,53]. The entire procedures were approved by Institutional Animal Care Unit Committee in Administration Office for Laboratory Animals, Beijing China (B10831).

\section{Dual recording}

The soma and axonal bleb of identical pyramidal neurons in layers IV-V of cerebral cortex were simultaneously recorded (MultiClapm-700B, Axon Instrument Inc. USA) under a fluorescent and DIC microscope (Nikon FN-E600; [14]. Electrical signals were inputted into pClamp-10 with $50 \mathrm{kHz}$ sampling rate. In wholecell recording, action potentials were induced by the signals recorded intracellularly in vivo. The judgment for recording two sites from an identical neuron is based on the synchronous presence of direct and corresponding electrical signals. Transient capacitance was compensated. Output bandwidth was $3 \mathrm{kHz}$. Pipette solution contains (mM) $150 \mathrm{~K}$-gluconate, $5 \mathrm{NaCl}, 0.4$ EGTA, 4 Mg-ATP, 0.5 Tris- GTP, 4 Na-phosphocreatine and 10 HEPES (pH 7.4 adjusted by $2 \mathrm{M} \mathrm{KOH}$ ). The osmolarity of pipette solution was 295-305 mOsmol. The pipette resistance was 10-15 $\mathrm{M} \Omega$. 
Neuronal intrinsic properties include spike thresholds (Vts) and refractory periods (RP). Vts were measured by depolarization pulses. RPs were measured by injecting two pulses (5\% above threshold) into neurons after each spike under current-clamp, in which inter-pulse intervals were adjusted $[12,13,25,54,55]$. The duration of pulses was $50 \mathrm{~ms}$, the minimal time period of in vivo signals (Figure 1C)

Latencies between axonal spikes and somatic ones, used to judge spike initiation, were measured based on the following thoughts. Elements in an electrical circuit of cell membrane includes voltage- gated conductance (Rv) for the generation of active signals, such as action potentials and synaptic signals, as well as passive membrane properties (input resistance, Rin; membrane capacitance, $\mathrm{Cm}$; inset in Figure 3A). We ruled out the effects of Rin and $\mathrm{Cm}$ on the analyses of temporal signals via subtracting the responses (gray lines in $3 \mathrm{~A}$ ) evoked by depolarization and hyperpolarization in the same intensities, such that spike potentials (black line in Figure 3A) were mediated by voltage-gated channels. The derivative of somatic and axonal spike potentials vs. time $(d v / d t)$ was calculated. The site of spike initiation was defined as a time point with a minimal $d v / d t$ but larger than zero (Figure 3B), which accurately represents the locus of spike initiation in the comparison with the peak, $50 \%$ rising phase or initial phase (onset point) of spikes [20-24,50,56-58]. Latencies between somatic spikes and axonal ones were the time difference of their initiation $(\Delta \mathrm{T}=\mathrm{T}$ soma-Taxon).

\section{In vivo recording}

Intracellular recordings with sharp electrodes were done at cortical pyramidal neurons from mice in vivo that were anesthetized by injecting chloral hydrate $(300 \mathrm{mg} /$ $\mathrm{kg}$ ). Electrical signals were recorded by an AxonClamp$2 \mathrm{~B}$ and sampled by pClamp-9 with $50 \mathrm{kHz}$ sampling rate (Axon Instrument Inc, USA). Electrodes were filled with $2 \mathrm{M}$ potassium acetate and their resistances were $50 \sim 70 \mathrm{M} \Omega$. The data were analyzed if resting membrane potentials were $-65 \mathrm{mV}$, and action potentials showed overshot [14].

\section{Computational Model}

The values of axonal and somatic Vts and ARP (Figure 3) were introduced into the axon and soma in NEURON (v7.0), respectively, to examine spikes' initiation. Other factors of VGSCs were based on Hodgkin-Huxley kinetics and current reports [9-11]. VGSCs' distributions in two compartments were based on the references [4,5,9-11]. VGSCs' reversal potential was $50 \mathrm{mV}$. For potassium channels, high-voltage-activated $\mathrm{K}^{+}$channels (Kv) and a slowly-activated/non-inactivated $\mathrm{M}$-type $\mathrm{K}^{+}$channels $(\mathrm{Km})$ were added into the modeling. To have the initiation of sequential spikes, the densities of $\mathrm{Kv}$ and $\mathrm{Km}$ were 50 and $100 \mathrm{pS} / \mathrm{cm}^{2}$ in the two compartments, respectively. Reversal potential for $\mathrm{Kv}$ was $-77 \mathrm{mV}$. In addition, cylinder axon was calculated based on $1.6 \mu \mathrm{m}$ in diameter and 70 $\mu \mathrm{m}$ in length, and the soma was $30 \mu \mathrm{m}$ in diameter. Their passive electrical properties include $C_{\mathrm{m}}=1 \mu \mathrm{F} / \mathrm{cm}^{2}, R_{\mathrm{m}}=$ $15000 \Omega / \mathrm{cm}^{2}$ and $R_{\mathrm{i}}=100 \Omega / \mathrm{cm}$. Resting membrane potentials were $-71 \mathrm{mV}$. The simulations were run under $37^{\circ} \mathrm{C}$. The time step was $0.025 \mathrm{~ms}$.

\section{Data analyses}

The data were analyzed if the soma and axon had resting membrane potentials negatively more than $-63 \mathrm{mV}$ and action potentials above $95 \mathrm{mV}$. Criteria for the acceptation of each experiment also included less than $5 \%$ changes in resting membrane potential, spike magnitude, input and seal resistance during each experiment. The values of Vts and RP are presented as mean $\pm \mathrm{SE}$. The comparisons between groups are done by paired t-test.

\section{Acknowledgements}

This study is granted by Natural Science Foundation China (30870517 and 30990261), National Basic Research Program (2011CB504405) and National Award for Outstanding Young Scientist (30325021) to JHW.

\section{Authors' contributions}

RG does to the experiments and data analysis, HQ works on computational simulation and JW contributes to experimental design and manuscript writing.

All authors have read and approved the final manuscript.

\section{Competing interests}

The authors declare that they have no competing interests.

Received: 16 March 2011 Accepted: 8 May 2011 Published: 8 May 2011

\section{References}

1. Kandel ER, Siegelbaum SA, Schwartz JH: Elementary interactions between neurons: Synaptic transmission. In Principles of Neural Science. Edited by: E. R. Kandel, Schwartz, J. H., and Jessell, T. M. McGraw-Hill: New York; 2000:175-308.

2. Shepherd GM: Electronic properties of axons and dendrites. In From Molecular to Networks: An Introduction to Cellular and Molecular Neuroscience. Edited by: J.H. Byrne and J.L. Roberts. New York: Elsevier Science (USA); 2004:91-113.

3. Brock LG, Coombs JS, Eccles JC: Intracellular recording from antidromically activated motoneurones. Journal of Physiology (London) 1953, 122:429-461.

4. Clark BA, et al: The site of action potential initiation in cerebellar Purkinje neurons. Nature Neuroscience 2005, 8(2):137-139.

5. Colbert CM, Pan E: lon channel properties underlying axonal action potential initiation in pyramidal neurons. Nature Neuroscience 2002, 5(6):533-538.

6. Eccles JC: The physiology of nerve cells. In The Physiology of Nerve Cells. Edited by: J.C. Eccles. Baltimore: Johns Hopkins University Press; 1957:1-50.

7. Edwards C, Ottoson D: The site of impulse initiation in a nerve cell of a crustacean stretch receptor. Journal of Physiology (London) 1958, 143:138-148.

8. Fuortes MGF, Frank K, Becker MC: Steps in the production of motor neuron spikes. Journal of General Physiology 1957, 40:735-752.

9. Hu W, et al: Distinct contribution of Nav1.6 and Nav1.2 in action potential initiation and backpropagation. Nature Neuroscience 2009, 12(8):996-1002. 
10. Kole MHP, et al: Action potential generation requires a high sodium channel density in the axon initial segment. Nature Neuroscience 2008 11(2):178-186.

11. Kole $M H$, Stuart GJ: Is action potential threshold lowest in the axon? Nat Neurosci 2008, 11(11):1253-5.

12. Chen N, Chen X, Wang JH: Homeostasis established by coordination of subcellular compartment plasticity improves spike encoding. Journal of Cell Science 2008, 121(17):2961-2971.

13. Wang $\mathrm{JH}$, et al: The gain and fidelity of transmission patterns at cortical excitatory unitary synapses improve spike encoding. Journal o $f \mathrm{Cell}$ Science 2008, 121(17):2951-2960.

14. Chen $\mathrm{N}$, et al: Axons amplify somatic incomplete spikes into uniform amplitudes in mouse cortical pyramidal neurons. PLOS ONE 2010, 5(7) e11868.

15. Deqenetais $E$, et al: Electrophysiological properties of pyramidal neurons in the rat prefrontal cortex: an in vivo intracellular recording study. Cerebral Cortex 2002, 12(1):1-16.

16. Haider $B$, et al: Neocortical network activity in vivo is generated through a dynamic balance of excitation and inhibition. Journal of Neuroscience 2006, 26(17):4535-4545.

17. Henze DA, Buzsaki G: Action potential threshold of hippocampal pyramidal cells in vivo is increased by recent spiking activity. Neuroscience 2001, 105(1):121-130.

18. Zhang $Z$, et al: Reprint of "frequency tuning and firing pattern properties of auditory thalamic neurons: an in vivo intracellular recording from the guinea pig". Neuroscience 2008, 154(1):273-282.

19. Fellous JM, et al: Frequency dependence of spike timing reliability in cortical pyramidal cells and interneuron. Journal of Neurophysiology 2001, 85:1782-1787.

20. Foust A, et al: Action potentials initiate in the axon initial segment and propagate through axon collaterals reliably in cerebellar Purkinje neurons. J Neurosci 2010, 30(20):6891-902

21. Palmer LM, Stuart GJ: Site of action potential initiation in layer 5 pyramidal neurons. J Neurosci 2006, 26(6):1854-63.

22. Palmer $L M$, et al: Initiation of simple and complex spikes in cerebellar Purkinje cells. J Physiol 2010, 588(Pt 10):1709-17.

23. Shu YS, et al: Properties of action potential initiation in neocortical pyramidal cells: Evidence from whole cell axon recordings. Journal Neurophysiology 2007, 97:746-760

24. Stuart GJ, Schiller J, Sakmann B: Action potential initiation and propagation in rat neocortical pyramidal neurons. Journal of Physiology (London) 1997, 505(Pt 3):617-632

25. Chen $\mathrm{N}$, et al: The refractory periods and threshold potentials of sequential spikes measured by whole-cell recordings. Biochemical and Biophysical Research Communications 2006, 340:151-157.

26. Mantegazza M, Franceschetti S, Avanzini G: Anemone toxin (ATX II)induced increase in persistent sodium current: effects on the firing properties of rat neocortical pyramidal neurones. J Physiol 1998, 507(Pt 1):105-16.

27. Hines ML, Carnevale NT: The NEURON simulation environment. Neural Computation 1997, 9(6):1179-1209.

28. Mainen ZF, et al: A model of spike initiation in neocortical pyramidal neurons. Neuron 1995, 15(6):1427-1439.

29. Chen WR, Midtgaard J, Shepherd GM: Forward and backward propagation of dendritic impulses and their synaptic control in mitral cells. Science 1997, 278:463-467.

30. Chen WR, et al: Multiple modes of action potential initiation and propagation in mitral cell primary dendrite. Journal of Neurophysiology 2002, 88(2):2755-2764

31. Gasparini S, Migliore M, Magee JC: On the initiation and propagation of dendritic spikes in CA1 pyramidal neurons. Journal of Neuroscience 2004 24(49):11046-11056.

32. Gulledge AT, Stuart GJ: Action potential initiation and propagation in layer 5 pyramidal neurons of the rat prefrontal cortex: absence of dopamine modulation. Journal of Neuroscience 2003, 23(36):11363-11372.

33. Hanson JE, Smith Y, Jaeger D: Sodium channels and dendritic spike initiation at excitatory synapses in globus pallidus neurons. Journal of Neuroscience 2004, 24(2):329-340.

34. Larkum ME, et al: Dendritic spikes in apical dendrites of neocortical layer 2/3 pyramidal neurons. Journal of Neuroscience 2007, 27(34):8999-9008.
35. Luscher HR, Larkum ME: Modeling action potential initiation and backpropagation in dendrites of cultured rat motoneurons. Journal of Neurophysiology 1998, 80(2):715-729.

36. Roberts $C B$, et al: Dendritic action potential initiation in hypothalamic gonadotropin-release hormone neurons. Endocrinology 2008, 149(7):3355-3360.

37. Angelides KJ, et al: Distribution and lateral mobility of voltage-dependent sodium channels in neurons. J Cell Biol 1988, 106(6):1911-25.

38. Boiko $T$, et al: Functional specialization of the axon initial segment by isoform-specific sodium channel targeting. J Neurosci 2003, 23(6):2306-13.

39. Duflocq A, et al: Nav1.1 is predominantly expressed in nodes of Ranvier and axon initial segments. Mol Cell Neurosci 2008, 39(2):180-92

40. Fleidervish $\mid \mathrm{A}$, et al: $\mathrm{Na}+$ imaging reveals little difference in action potential-evoked $\mathrm{Na}+$ influx between axon and soma. Nature Neuroscience 2010, 13(7):852-860.

41. Garrido JJ, et al: Dynamic compartmentalization of the voltage-gated sodium channels in axons. Biol Cell 2003, 95(7):437-45.

42. Hill AS, et al: Ion channel clustering at the axon initial segment and node of Ranvier evolved sequentially in early chordates. PLoS Genet 2008, 4(12):e1000317.

43. Inda MC, Defelipe J, Munoz A: Voltage-gated ion channels in the axon initial segment of human cortical pyramidal cells and their relationship with chandelie cells. Proceeding in National Academy of Science USA 2006, 103:2920-2925

44. Lorincz A, Nusser Z: Cell-type-dependent molecular composition of the axon initial segment. J Neurosci 2008, 28(53):14329-40

45. Royeck $M$, et al: Role of axonal NaV1.6 sodium channels in action potential initiation of CA1 pyramidal neurons. J Neurophysiol 2008 100(4):2361-80

46. Schmidt-Hieber $C$, Bischofberger J: Fast sodium channel gating supports localized and efficient axonal action potential initiation. J Neurosci 2010 30(30):10233-42

47. Van Wart A, Trimmer JS, Matthews G: Polarized distribution of ion channels within microdomains of the axon initial segment. J Comp Neurol 2007, 500(2):339-52.

48. Wollner DA, Catterall WA: Localization of sodium channels in axon hillocks and initial segments of retinal ganglion cells. Proc Natl Acad SCi USA 1986, 83(21):8424-8.

49. Colbert CM, Johnston D: Axonal action-potential initiation and $\mathrm{Na}+$ channel densities in the soma and axon initial segment of subicular pyramidal neurons. J Neurosci 1996, 16(21):6676-86.

50. Kress GJ, et al: High threshold, proximal initiation, and slow conduction velocity of action potentials in dentate granule neuron mossy fibers. $J$ Neurophysiol 2008, 100(1):281-91.

51. Somogyi $P$, Klausberger $T$ : Defined types of cortical interneurone structure space and spike timing in the hippocampus. Journal of Physiology (London) 2005, 562(1):9-29.

52. $\mathrm{Ni} \mathrm{H}$, et al: Upregulation of barrel GABAergic neurons is associated with cross-modal plasticity in olfactory deficit. PLOS ONE 2010, 5(10):e13736.

53. Wang J-H: Short-term cerebral ischemia causes the dysfunction of interneurons and more excitation of pyramidal neurons. Brain Research Bulletin 2003, 60(1-2):53-58.

54. Chen $\mathrm{N}$, et al: After-hyperpolarization improves spike programming through lowering threshold potentials and refractory periods mediated by voltage-gated sodium channels. Biochemical and Biophysical Research Communications 2006, 346:938-945.

55. Chen N, et al: Sodium channel-mediated intrinsic mechanisms underlying the differences of spike programming among GABAergic neurons. Biochemical and Biophysical Research Communications 2006, 346:281-287

56. Kole MH, Letzkus JJ, Stuart GJ: Axon initial segment Kv1 channels control axonal action potential waveform and synaptic efficacy. Neuron 2007 55(4):633-47.

57. McCormick DA, Shu Y, Yu Y: Neurophysiology: Hodgkin and Huxley model-still standing? Nature 2007, 445(7123):E1-2, discussion E2-3.

58. Naundorf $B$, Wolf $F$, Volgushev M: Unique features of action potential initiation in cortical neurons. Nature 2006, 440(7087):1060-3.

doi:10.1186/1756-6606-4-19

Cite this article as: Ge et al:: Physiological synaptic signals initiate sequential spikes at soma of cortical pyramidal neurons. Molecular Brain 2011 4:19. 